\title{
Aspectos terapêuticos da esporotricose felina
}

\author{
Therapeutic aspects of feline sporotrichosis \\ Sandro Antonio Pereira', Tânia Maria Pacheco Schubach', Isabella Dib Ferreira Gremião', \\ Denise Torres da Silva', Fabiano Borges Figueiredo', \\ Núbia Vieira de Assis² \& Sônia Regina Lambert Passos ${ }^{3}$
}

\begin{abstract}
RESUMO
A esporotricose é uma micose subcutânea causada pelo fungo dimórfico Sporothrix schenckii que acomete seres humanos e uma grande variedade de animais. O gato é a espécie animal com a maior frequência de casos até o momento. Essa micose é frequente no Brasil, principalmente no Rio de Janeiro, e a transmissão zoonótica relacionada a gatos infectados vem aumentando nas duas últimas décadas. A esporotricose felina apresenta variadas apresentações clínicas e o tratamento representa um desafio para o médico veterinário. $\mathrm{O}$ itraconazol e o cetoconazol são os antifúngicos mais utilizados no tratamento da esporotricose felina, sendo o primeiro o fármaco de eleição. Os iodetos, a terbinafina, a termoterapia local, a anfotericina B e a remoção cirúrgica das lesões cutâneas são outras opções de tratamento. Diversos estudos enfocam os aspectos terapêuticos da esporotricose em seres humanos, entretanto, na esporotricose felina, existem poucos relatos ou estudos de séries de casos. Este artigo teve como objetivo descrever os esquemas terapêuticos atualmente utilizados no tratamento da esporotricose felina.
\end{abstract}

Descritores: esporotricose, Sporothrix schenckii, gatos, tratamento.

\begin{abstract}
Sporotrichosis is a subcutaneous mycosis caused by the dimorphic fungus Sporothrix schenckii. It affects human beings and a number of other animal species. Cats show the highest incidence and cat-related disease transmission to humans has been rising in the last twenty years in Brazil. Feline sporotrichosis has various clinical presentations and its treatment is a challenge to veterinarians. Itraconazole, as a first choice, and ketoconazole are the most commonly used medications. Iodides, terbinafine, local thermotherapy, amphothericin B and surgical resection of cutaneous lesions are other therapeutic options. There are several studies on therapeutic aspects of human sporotrichosis but a much smaller number for feline sporotrichosis. This paper describes the currently used therapeutic schemes for feline sporotrichosis treatment.
\end{abstract}

Keywords: sporotrichosis, Sporothrix schenckii, cats, treatment. 


\section{INTRODUÇ̃̃̃O}

\section{ESPOROTRICOSE FELINA}

\section{FÁRMACOS UTILIZADOS NO TRATAMENTO DA ESPOROTRICOSE FELINA}

\section{IODETOS}

\section{AZÓLICOS}

\subsection{CETOCONAZOL}

\subsection{ITRACONAZOL}

\section{TERBINAFINA}

\section{ANFOTERICINA B}

\section{OUTRAS MODALIDADES DE TRATAMENTO}

\section{TRATAMENTO CIRÚRGICO ASSOCIADO AO USO DE ITRACONAZOL}

\section{TERMOTERAPIA LOCAL}

\section{CONCLUSÕES}

\section{INTRODUÇÃO}

A esporotricose é causada pelo fungo Sporothrix schenckii, que acomete humanos e animais [70], sendo o gato o animal que apresenta a maior frequência de casos $[64,77]$. Geralmente é adquirida através da pele pela implantação traumática do fungo presente em matéria orgânica, solo e plantas [70]. Embora menos frequentes, as formas pulmonar e disseminada podem ser adquiridas através da inalação de conídios [75].

Sporothrix schenckii é um fungo dimórfico, que existe nas formas de hifa a $25^{\circ} \mathrm{C}$ e de levedura a $37^{\circ} \mathrm{C}$. O diagnóstico definitivo da esporotricose requer o isolamento de $S$. schenckii em cultura, inicialmente na forma filamentosa, e posterior termoconversão à fase leveduriforme [70].

A esporotricose é frequente no Brasil, onde o maior número de casos é proveniente do Rio de Janeiro $[3,64,78]$. Nos últimos anos, os relatos de transmissão zoonótica vem aumentando nessa região [76].

Os iodetos, os azólicos, a anfotericina $\mathrm{B}$, a terbinafina, a termoterapia local ou remoção cirúrgica das lesões são opções disponíveis para o tratamento da esporotricose felina $[30,31,39,77]$.

O tratamento da esporotricose felina representa um desafio para o médico veterinário [87]. A cura, a falência terapêutica e os efeitos adversos ocorrem independentemente do esquema terapêutico utilizado $[64,77]$.

Recentemente, foram estabelecidas recomendações baseadas em evidências para o tratamento da esporotricose humana [44]. Entretanto, na esporotricose felina, foram encontrados poucos relatos ou estudos de séries de casos [77].

Este artigo tem como objetivo descrever os esquemas terapêuticos atualmente utilizados no tratamento da esporotricose felina.

\section{ESPOROTRICOSE FELINA}

A susceptibilidade experimental do gato a $S$. schenckii foi descrita no início do século XX [5]. Entretanto, o primeiro relato de infecção naturalmente adquirida ocorreu em 1952 [83], seguida da primeira descrição no Brasil [26]. Até a década de 1990, existiam poucos relatos da doença em gatos no Brasil [77].

A infecção por $S$. schenckii em gatos pode iniciar de forma subclínica e evoluir para lesões cutâneas múltiplas e comprometimento sistêmico fatal, associado ou não a sinais extracutâneos, principalmente respiratórios [77].

As lesões cutâneas mais frequentes em gatos são nódulos, gomas e úlceras recobertas ou não por crostas, que podem evoluir até necrose com exposição de músculos e ossos [80]. A maioria dessas lesões está localizada na cabeça, nas extremidades dos membros e na cauda [73].

Nos gatos infectados por S. schenckii, geralmente observa-se uma grande quantidade de leveduras nos tecidos [79] e no exsudato de lesões cutâneas ou mucosas [82]. 
Aproximadamente $40 \%$ dos gatos com esporotricose assistidos no Instituto de Pesquisa Clínica Evandro Chagas (IPEC)/Fundação Oswaldo Cruz (FIOCRUZ) - Rio de Janeiro, RJ, apresentaram lesões cutâneas em três ou mais localizações não contíguas [64,77]. Linfadenite, linfangite nodular ascendente e lesões mucosas podem estar presentes [77,82].

Sinais respiratórios foram observados em $44 \%$ dos gatos com esporotricose [77]. Em um estudo realizado por Pereira et al. [64], a presença desses sinais foi inversamente associada à cura clínica e diretamente associada ao óbito, com risco cerca de duas vezes maior. Neste estudo, o espirro foi o sinal respiratório mais frequente, ocorrendo em aproximadamente $40 \%$ dos casos.

As diferentes apresentações clínicas da esporotricose felina podem influenciar no prognóstico e consequentemente, no desfecho do quadro $[64,77]$.

Não se sabe ainda porque os gatos, diferentemente dos seres humanos e dos outros animais, apresentam uma maior sensibilidade ao $S$. schenckii [19]. Imunodepressão generalizada ou a ocorrência de anergia específica ao $S$. schenckii foram sugeridas [6].

$\mathrm{O}$ uso de fármacos imunossupressores é contraindicado durante e após o tratamento da esporotricose felina [73].

Para alguns autores, existe uma associação entre a ocorrência de infecções fúngicas em gatos e a coinfecção com o vírus da Imunodeficiência Felina (FIV) ou com vírus da Leucemia Felina (FeLV) $[22,52]$. Outros autores não encontraram associação entre a gravidade da infecção fúngica e a coinfecção com esses retrovírus [14].

Schubach et al. [77] testaram 142 gatos para detecção de anticorpos antiFIV e antígenos do FeLV e observaram aproximadamente $20 \%$ dos gatos positivos para FIV, $1 \%$ de positivos para FeLV e $1 \%$ de positividade para ambos. Esses autores relataram a cura clínica de oito gatos com anticorpos anti-FIV e não observaram diferenças na resposta ao tratamento entre animais coinfectados ou não. Pereira et al. [64] testaram 46 gatos e detectaram nove com anticorpos antiFIV, dos quais oito responderam ao tratamento. Entretanto, o pequeno número de gatos testados não permitiu inferir se a coinfecção pelo FIV interferiu no curso do tratamento.

A cura espontânea de gatos infectados por $S$. schenckii foi descrita, entretanto é rara $[48,77]$. Na maioria dos casos de esporotricose felina, há necessidade de tratamento.

Os dois maiores estudos sobre terapêutica da esporotricose felina foram realizados no Rio de Janeiro - RJ, Brasil [64,77].

Schubach et al. [77] avaliaram diferentes esquemas terapêuticos em 266 gatos doentes. A cura clínica foi obtida em $25,4 \%$, a duração do tratamento variou de 16 a 80 semanas (mediana $=36$ semanas) e os efeitos adversos mais observados foram anorexia, vômito e diarreia.

Pereira et al. [64] compararam a eficácia e a segurança do tratamento por via oral com cetoconazol ou itraconazol em 773 gatos com esporotricose no período de 2002 a 2005, dos quais: $30,8 \%$ curaram-se, $13,6 \%$ foram a óbito por diferentes causas e $55,6 \%$ abandonaram ou ainda se encontravam em tratamento. Efeitos adversos ocorreram em 39,6\% dos gatos, sendo a hiporexia o mais frequente $(31,3 \%)$.

A adesão à prescrição pode interferir no prognóstico e no desfecho do caso [64,77]. A dificuldade de administração dos fármacos por via oral ao gato, o tratamento por longo período e a aquisição da doença por um membro da família são fatores que levam ao frequente abandono do tratamento e à requisição de eutanásia pelo responsável pelo gato [77].

Devido ao aumento de relatos da esporotricose felina em nosso meio nos últimos anos [13,21,30,39, $41,50,60,64,65,77,82]$, o conhecimento sobre os agentes antifúngicos e outras formas de tratamento é necessário para prescrição e acompanhamento terapêutico dos pacientes.

\section{FÁRMACOS UTILIZADOS NO TRATAMENTO DA ESPOROTRICOSE FELINA}

\section{Iodetos}

O tratamento da esporotricose teve início em 1903, quando De Beurmann e Ramond, seguindo sugestão de Sabouraud, empregaram o iodeto de potássio em seres humanos [15]. Esse composto foi considerado como terapia de eleição durante vários anos [45].

O mecanismo de ação dos iodetos ainda é desconhecido [43]. A ação direta dos compostos iodados [86] ou a ativação de macrófagos pelo iodo poderiam ser responsáveis pela cicatrização [28]. Autores sugerem que o iodo aumenta a resposta imune, entretanto, o mecanismo através do qual isso ocorre não foi ainda esclarecido [53]. 
A dose recomendada para o tratamento da esporotricose felina varia de $10-20 \mathrm{mg} / \mathrm{kg}$ a cada 12 ou $24 \mathrm{~h}$, por via oral $[29,77]$.

Schubach et al. [77] relataram a cura clínica de seis gatos com esporotricose utilizando iodeto de sódio por via oral na dose de $10 \mathrm{mg} / \mathrm{kg}$ a cada $12 \mathrm{~h}$. De todos os pacientes que receberam esse fármaco $40,5 \%$ apresentaram efeitos adversos.

Os felinos são muito sensíveis aos iodetos [20]. Depressão, anorexia, vômito, espasmos musculares, hipotermia, colapso cardiovascular, ptialismo, hiperexcitabilidade, pelagem seca, diarreia, hipertermia e cardiomiopatia são os sinais mais frequentes de iodismo [7,61].

Os gatos podem não responder ao tratamento com iodetos, e evoluir com piora do quadro clínico $[60,62]$ e óbito $[1,26,60]$. A substituição dos iodetos pelos azóis é recomendada quando forem observados efeitos adversos graves, buscando maior efetividade e segurança [87].

\section{Azólicos}

\subsection{Cetoconazol}

Em 1979, iniciaram-se os estudos de fase II com o cetoconazol para o tratamento de micoses incluindo a esporotricose. As suas vantagens sobre os fármacos existentes residiam na menor toxicidade, na fácil administração por longo período e no amplo espectro de atividade in vivo e in vitro [18]. A atividade é primariamente fungistática, podendo ser fungicida em doses elevadas [61]. O principal efeito dos azólicos sobre os fungos é a inibição da $14-\alpha$-esterol demetilase, prejudicando a biossíntese do ergosterol, componente vital da membrana fúngica [4].

Requer um $\mathrm{pH}$ ácido para absorção oral máxima [85] e a ligação às proteínas é alta (>98\%). É bem distribuído na pele e no tecido celular subcutâneo, entretanto, não penetra adequadamente nos fluidos cérebro-espinhal, seminal e ocular. A biotransformação é hepática e a excreção ocorre principalmente através da bile. A meia-vida de eliminação é de aproximadamente 10 horas, sendo dose-dependente [34].

O cetoconazol está disponível no Brasil na forma de comprimidos de $200 \mathrm{mg}$. Atualmente existe um produto de uso veterinário disponível no mercado na forma de solução oral a $20 \%$.

A experiência com o cetoconazol no tratamento da esporotricose em seres humanos é limitada e os resultados obtidos são variados $[9,18,59,74]$. Na medicina veterinária, esse fármaco vem sendo utilizado com frequência [57], embora existam poucos relatos de tratamento da esporotricose felina $[7,55,60$, 64,77].

Foi descrito o êxito do tratamento da esporotricose felina com a combinação sequencial de cetoconazol $5-10 \mathrm{mg} / \mathrm{kg} /$ dia seguido por iodeto de sódio $20-40 \mathrm{mg} / \mathrm{kg} / \mathrm{dia}$ [7]. Schubach et al. [77] relataram sucesso com cetoconazol $5-10 \mathrm{mg} / \mathrm{kg} /$ dia no tratamento da esporotricose felina. Entretanto, existem relatos de insucesso do cetoconazol $10 \mathrm{mg} / \mathrm{kg} / \mathrm{dia}$ no tratamento de gatos com esporotricose $[55,60]$. Em um estudo conduzido por Pereira et al. [64], o cetoconazol 50-100mg a cada 12 ou $24 \mathrm{~h}(13,5-27,0 \mathrm{mg} / \mathrm{kg} / \mathrm{dia})$ foi empregado em 598 gatos. A cura clínica foi observada em $28,6 \%$ dos casos num tempo mediano de tratamento de 28 semanas e em $42,1 \%$ dos casos, houve a ocorrência de efeitos adversos gastrintestinais.

$\mathrm{O}$ fármaco apresenta baixa toxicidade seletiva sobre o citocromo $\mathrm{P} 450$ do fungo, com ação sobre o citocromo P450 de mamíferos, o que resulta nos seguintes efeitos adversos: vômitos, anorexia e náuseas, com aumento freqüente das enzimas hepáticas [34]. O fracionamento da dose, com administração do fármaco a cada 12 horas, ou a redução da mesma, podem ser benéficas nos casos de ocorrência de efeitos adversos gastrintestinais dose-dependentes [85]. Os gatos, quando comparados com os cães, podem apresentar elevada toxicidade hepática [88], sendo recomendado o monitoramento periódico das enzimas hepáticas [85].

O cetoconazol é teratogênico e embriotóxico em ratas. Apresenta interações com diversos fármacos, como antiácidos e prednisolona [68], portanto, recomenda-se cautela antes de prescrever o cetoconazol simultaneamente com outros fármacos [16].

Ultimamente, o cetoconazol tem sido substituído pelo derivado triazólico itraconazol no tratamento das micoses, exceto quando o baixo custo do primeiro superar as vantagens do segundo [4].

\subsection{Itraconazol}

Desde a década de 1980, o itraconazol tem sido utilizado para o tratamento de micoses superficiais, subcutâneas e em infecções fúngicas sistêmicas [4], inclusive no tratamento da esporotricose $[24,69]$. Em medicina veterinária, é utilizado com sucesso no tratamento de diversas micoses em gatos [29]. É considerado o fármaco de eleição nos casos de esporotricose 
humana [44] e felina [87], devido à sua eficácia e segurança em comparação com os demais antifúngicos [84]. Entretanto, o custo é um fator limitante nos países em desenvolvimento [2].

O itraconazol é 5-100 vezes mais potente in vitro e in vivo do que o cetoconazol, embora o mecanismo de ação seja similar [34]. Apresenta atividade fungistática, mas pode ter ação fungicida, dependendo da concentração administrada e da susceptibilidade do fungo [68].

A absorção do itraconazol, por via oral, é altamente dependente do $\mathrm{pH}$ gástrico, sendo recomendada a sua administração com refeições [8], pois aumenta de duas a três vezes a biodisponibilidade deste fármaco [85]. A ligação às proteínas plasmáticas é alta ( $>99 \%)$. Devido à afinidade lipofílica e protéica, apresenta ampla distribuição na maioria dos tecidos orgânicos, em que as concentrações da substância são superiores às encontradas no plasma, explicando a sua eficácia no tratamento de diferentes micoses $[8,10]$. Contudo, não é bem distribuído em fluidos orgânicos tais como: líquor, fluidos oculares e saliva [34].

O metabolismo é hepático e a eliminição, predominantemente pela via biliar [68], porém alguns de seus metabólitos são encontrados na urina [34]. Apresenta alta seletividade às enzimas do citocromo $\mathrm{P} 450$ fúngico em relação aos azólicos conhecidos, resultando em menor ocorrência de efeitos adversos [34].

$\mathrm{O}$ itraconazol está disponível em cápsulas e soluções para uso oral e administração intravenosa [4]. No Brasil, é encontrado somente na forma de cápsulas de $10 \mathrm{mg}, 25 \mathrm{mg}, 50 \mathrm{mg}$ e $100 \mathrm{mg}$.

Estudos relatando a utilização do itraconazol na terapêutica da esporotricose felina são escassos [77].

A dose de itraconazol classicamente recomendada para o tratamento da esporotricose felina é $5-10 \mathrm{mg} / \mathrm{kg}$ a cada 12-24h [73]. O tempo de tratamento é prolongado e a administração do fármaco deve continuar minimamente um mês após a cura clínica [87]. Autores descreveram êxito no tratamento da esporotricose felina utilizando o itraconazol $5-10 \mathrm{mg} / \mathrm{kg} / \mathrm{dia}$ [41,50,77]. Pereira et al. [64] relataram a cura clínica em 38,3\% (67/175) dos gatos tratados com itraconazol $30-100 \mathrm{mg}$ a cada 12 ou $24 \mathrm{~h}(8,3-27,7 \mathrm{mg} / \mathrm{kg} / \mathrm{dia})$. O tempo mediano de tratamento até a cura clinica foi de 26 semanas e 30,9\% (54/175) dos gatos apresentaram efeitos adversos gastrintestinais. Esses mesmos autores concluíram que o itraconazol apresentou superioridade, se comparado ao cetoconazol no tratamento da esporotricose felina.

A frequência de efeitos adversos gastrintestinais em gatos tratados com itraconazol é inferior à relatada com cetoconazol [64]. As mesmas medidas preconizadas para o cetoconazol, quando ocorrem esses efeitos, podem ser aplicadas para o itraconazol, inclusive o monitoramento seriado das enzimas hepáticas. Na ausência de sinais clínicos de hepatotoxicidade, as concentrações enzimáticas tendem a voltar ao normal após a suspensão do fármaco [85].

Em animais de laboratório, o itraconazol em doses elevadas, causa toxicidade fetal e teratogenia [68].

Como no cetoconazol, as interações ocorrem com um grande número de fármacos, portanto recomenda-se cuidado na administração simultânea desses com o itraconazol [16].

\section{Terbinafina}

A terbinafina é um antifúngico pertencente à classe das alilaminas, que tem sido efetivo no tratamento das dermatofitoses e de infecções superficiais causadas por leveduras em cães e gatos [29]. Tem mostrado alta atividade in vitro contra isolados de $S$. schenckii $[27,46,54,58]$. Entretanto, o potencial terapêutico tem sido confirmado somente para esporotricose cutânea e linfocutânea em seres humanos [11, $25,33,40,66]$.

Apresenta atividade fungicida contra dermatófitos [16] e sua ação resulta da inibição da esqualeno epoxidase fúngica, com bloqueio da síntese do ergosterol [4], independente do citocromo P450 [68].

É bem absorvida por via oral e a ingestão de alimentos pode aumentar discretamente sua absorção [68]. Apresenta alta ligação às proteínas plasmáticas e elevada lipossolubilidade, sendo bem distribuída no estrato córneo, nas glândulas sebáceas e nos folículos pilosos [71]. A metabolização é hepática, sendo os metabólitos inativos excretados pela urina [71]. A meiavida de eliminação é de aproximadamente 36 horas [68].

Está disponível no Brasil em comprimidos de $125 \mathrm{mg}$ e $250 \mathrm{mg}$.

Devido à experiência limitada em medicina veterinária, os efeitos adversos em animais ainda não estão bem estabelecidos, entretanto, efeitos adversos gastrintestinais podem ocorrer [68]. Estudos de 
toxicidade fetal em animais não evidenciaram reações adversas e as interações medicamentosas ocorrem em menor número quando comparado aos azóis [16].

Schubach et al. [77] relataram sucesso na utilização oral de terbinafina $30 \mathrm{mg}$ a cada $24 \mathrm{~h}$, no tratamento da esporotricose felina, isoladamente ou associado ao itraconazol, com efetividade de $7,4 \%$ e $5,9 \%$ dos casos, respectivamente.

\section{Anfotericina B}

A anfotericina B é um antibiótico macrolídeo poliênico obtido do actinomiceto Streptomyces nodosus [71]. Foi descoberta na década de 1950 e, em 1965, foi o primeiro antifúngico aprovado pelo FDA $[17,23]$. Tem sido indicada para o tratamento de micoses sistêmicas [71] e esporotricose disseminada em seres humanos [44].

A ação fungicida ou fungistática é dose-dependente. Em baixas concentrações, liga-se de forma reversível à membrana do fungo, provoca aumento da permeabilidade da mesma para íons de potássio e causa o efeito fungistático. Em doses elevadas, sua ligação à membrana do fungo requer gasto de energia, é irreversível e ocasiona a morte celular através de alterações das propriedades físico-químicas dessa membrana $[47,67]$.

A despeito da maior afinidade pelo ergosterol, alguns efeitos tóxicos são resultados da sua capacidade em ligar-se ao colesterol da membrana celular de mamíferos [71].

Além do efeito antifúngico, apresenta ação imunoestimulante, aumenta a resistência do hospedeiro à infecção e pode incrementar as propriedades fungicidas dos macrófagos [23,47].

A anfotericina B é pouco solúvel [23]. Sua formulação convencional é associada ao desoxicolato de sódio, cuja finalidade é solubilizá-la em água e estabilizar a suspensão na forma de micélios [56].

As doses de anfotericina B descritas na literatura médico-veterinária são variadas. Em um protocolo para tratamento de micoses em cães e gatos, recomendou-se a dose total de $4-12 \mathrm{mg} / \mathrm{kg}$ fracionada em 0,15-05 mg/kg/dia, por via intravenosa (IV), em dias alternados. Em casos refratários, doses diárias de $1 \mathrm{mg} / \mathrm{kg}$ foram administradas duas ou três vezes por semana. Em caso de associação com outros fármacos, deve-se utilizar a dose total mais baixa [34].

Doses superiores a $1 \mathrm{mg} / \mathrm{kg} /$ dia são mais efetivas, porém seu uso é limitado pelos efeitos tóxicos.
Para contornar esses problemas, foram desenvolvidas formulações lipídicas menos tóxicas. Três dessas formulações estão disponíveis: lipossomal, dispersão coloidal e complexo lipídico $[23,56]$. Porém, o custo do tratamento com as formulações lipídicas é alto, limitando o uso [23].

Foram descritos três casos de esporotricose felina tratados sem sucesso com anfotericina B por via IV, em virtude da ocorrência de efeitos adversos e óbitos $[19,49,62]$.

Em casos de esporotricose felina com falência terapêutica ao itraconazol, a associação de anfotericina B desoxicolato, por via subcutânea ou intralesional pode ser uma alternativa [31,72]. A associação itraconazol oral e anfotericina $\mathrm{B} 0,5 \mathrm{mg} / \mathrm{kg}$ por via subcutânea foi utilizada no tratamento de 17 gatos com esporotricose refratária ao tratamento com azólicos. A cura clínica ocorreu em seis casos e a formação de abscesso estéril local ocorreu em quatro [72]. Gremião et al. [31] obtiveram sucesso após associação de três aplicações de anfotericina B por via intralesional $(5 \mathrm{mg} /$ aplicação) e itraconazol em um gato com lesão cutânea persistente na ponte nasal após nove meses de tratamento com o mesmo azólico.

$\mathrm{O}$ tratamento intravenoso com anfotericina $\mathrm{B}$ quase sempre resulta em algum grau de disfunção renal [23], geralmente reversível após a interrupção [12]. A anemia decorrente da inibição da produção de eritropoetina pelos rins ou por ação direta do fármaco sobre a medula óssea é frequente [56], entretanto, em raras ocasiões, pode ocorrer trombocitopenia [10]. Outros efeitos adversos incluem arritmias cardíacas, falência hepática aguda, anafilaxia, distúrbios eletrolíticos, febre, calafrios, flebite e náuseas [34]. Esse fármaco pode ser utilizado durante a gravidez, em cadelas e gatas [63].

Além da nefrotoxicidade, sua utilização em animais é limitada pela dificuldade na administração, que requer: diluição em grande volume de solução glicosada a $5 \%$ e infusão intravenosa lenta, em dias alternados ou três vezes por semana, durante várias semanas $[51,84]$.

\section{OUTRAS MODALIDADES DE TRATAMENTO}

\section{Tratamento cirúrgico associado ao uso de itraconazol}

A excisão cirúrgica de lesão e a posterior utilização de itraconazol foi descrita em gatos com esporotricose $[13,35]$. 
A ressecção cirúrgica das lesões residuais após falência do tratamento medicamentoso foi relatada em ser humano e gato [30,42,81]. Gremião et al. [30] relataram sucesso na remoção cirúrgica de uma lesão persistente na bolsa escrotal e testículos de um gato com esporotricose em tratamento com itraconazol $20 \mathrm{mg} / \mathrm{kg} / \mathrm{dia}$.

A principal limitação desta modalidade de tratamento é que a lesão deve estar localizada em um sítio anatômico que permita a intervenção cirúrgica [42].

\section{Termoterapia local}

$\mathrm{Na}$ esporotricose em seres humanos, a hipertermia local é usada como uma alternativa no tratamento das formas cutânea e cutâneo-linfática [32,38]. O crescimento de $S$. schenckii é inibido quando a temperatura é igual ou maior que $40^{\circ} \mathrm{C}[36,37]$. Esse método é recomendado em grávidas e pacientes com intolerância aos iodetos, aos azólicos e à terbinafina [8].

Honse et al. [39] relataram a cura clínica de um gato com esporotricose apresentando lesão cutânea única, utilizando somente a termoterapia local, duas vezes ao dia, durante sete semanas.

As limitações desse tratamento no gato envolvem a localização das lesões, determinação do momento correto para suspensão da terapia e a dificuldade na determinação da temperatura adequada [8,38]. Adicionalmente, é necessário que o paciente seja cooperativo e que apresente a forma fixa da esporotricose [39].

\section{CONCLUSÕES}

Estudos terapêuticos sobre a esporotricose felina são escassos e limitados a relatos e séries de casos.

As opções terapêuticas disponíveis para o tratamento da esporotricose felina permanecem limitadas pelo número de fármacos disponíveis e pela dificuldade do manejo do gato.

Os azólicos, particularmente o itraconazol, são os fármacos mais usados no tratamento. Houve uma variação importante no limite superior da dose desse grupo farmacológico.

A terbinafina é efetiva e segura nos casos humanos de esporotricose nas formas cutânea e linfocutânea, entretanto, na esporotricose felina esses parâmetros ainda não são bem conhecidos.

A anfotericina B associada ao itraconazol é recomendada nas formas sistêmica e extracutânea da esporotricose em humanos, no entanto, há pouca informação sobre efetividade e segurança desse esquema terapêutico na esporotricose felina.

Ensaios clínicos randomizados, duplocegos e controlados com diferentes agentes antifúngicos são necessários para determinação de esquemas terapêuticos mais eficazes e seguros para o tratamento da esporotricose felina.

Agradecimentos. Aos pesquisadores Armando de Oliveira Schubach e Raquel Vasconcellos Carvalhaes de Oliveira, professores do Programa de Pós-graduação em Pesquisa Clínica em Doenças Infecciosas do IPEC/FIOCRUZ, pela colaboração na elaboração final deste artigo.

\section{REFERÊNCIAS}

1 Anderson N.V., Ivoghii D., Moore W.E. \& Leipold H.W. 1973. Cutaneous sporotrichosis in a cat: A case report. Journal of the American Animal Hospital Association. 9: 526-529.

2 Barros M.B.L. 2004. Estudo de uma série de casos de esporotricose atendidos no Instituto de Pesquisa Clínica Evandro Chagas, Rio de Janeiro. 138f. Rio de Janeiro, RJ. Tese (Doutorado em Doenças Infecciosas e Parasitárias) - Programa de Pós-graduação em Medicina, Universidade Federal do Rio de Janeiro.

3 Barros M.B.L., Schubach A., Francesconi-do-ValleA.C., Gutierrez Galhardo M.C., Conceição-Silva F., Schubach T.M.P., Reis R.S., Marzochi K.B.F., Wanke B. \& Conceição M.J. 2004. Cat-transmitted sporotrichosis epidemic in Rio de Janeiro, Brazil: description of a series of cases. Clinical Infectious Diseases. 38: 529-535.

4 Bennet J.E. 2006. Antimicrobial agents: antifungal agents. In: Hardman J.G., Limbird L.E. \& Gilman A.G. (Eds). Goodman \& Gilman's The pharmacological basis of therapeutics. New York: McGraw-Hill, pp.1225-1242.

5 Beurmann L. \& Gougerot H. 1906. Les sporotrichoses hypodermiques. Annales de Dermatologie et de Syphiligraphie. 7: 993-1006.

6 Bickley L.K., Berman I.J. \& Hood A.F. 1985. Fixed cutaneous sporotrichosis: unusual histopathology following intralesional corticosteroid administration. Journal of the American Academy of Dermatology. 12: 1007-1012.

7 Burke M., Grauer G. \& Macy D. 1982. Successful treatment of cutaneolymphatic sporotrichosis in cat with ketoconazole and sodium iodine. Journal of the American Animal Hospital Association. 19: 542-547. 
8 Bustamante B. \& Campos P.E. 2004. Sporotrichosis: a forgotten disease in the drug research agenda. Expert Review of AntiInfective Therapy. 2: 85-94.

9 Calhoun D.L., Waskin H., White M.P., Bonner J.R., Mulholland J.H., Rumans L.W., Stevens D.A. \& Galgiani J.N. 1991. Treatment of systemic sporotrichosis with ketoconazole. Reviews of Infectious Diseases. 13: 47-51.

10 Catalán M. \& Montejo J.C. 2006. Antifúngicos sistémicos. Revista Iberoamericana de Micologia. 23: 39-49.

11 Chapman S.W., Pappas P., Kauffmann C., Smith E.B., Dietze R., Tiraboschi-Foss N., Restrepo A., Bustamante A.B., Opper C., Emady-Azar S. \& Bakshi R. 2004. Comparative evaluation of the efficacy and safety of two doses of terbinafine (500 and $1000 \mathrm{mg}$ day) in the treatment of cutaneous or lymphocutaneous sporotrichosis. Mycoses. 47: 62-68.

12 Coraçari A.R., Alves A.T., Brasil R.G., Fernandes A.M. \& Maniglia J.V. 2003. Mucormicose rino-órbito-cerebral: relato de caso e revisão de literatura. Arquivos Internacionais de Otorrinolaringologia. 7:160-165 [Fonte: <http:/www.arquivosdeorl.org.br/ conteudo/acervo_port.asp?id=234>].

13 Corgozinho K.B., Souza H.J.M., Neves A., Fusco M.A. \& Belchior C. 2006. Um caso atípico de esporotricose felina. Acta Scientiae Veterinariae. 34: 167-170.

14 Davies C. \& Troy G.C. 1996. Deep mycotic infections in cats. Journal of the American Animal Hospital Association. 32 : 380-391.

15 De Beurmann L. \& Ramond. 1903. Abcès sous-cutanés multiples d'origine mycosique. Annales de Dermatologie et de Syphiligraphie. 4: 678-685.

16 de Jaham C., Paradis M. \& Papich M. 2000. Antifungal dermatologic agents: azoles and allylamines. Small Animal / Exotics. 22(6): 548-559.

17 Dismukes W.E. 2000. Introduction to antifungal drugs. Clinical Infectious Diseases. 30: 653-657.

18 Dismukes W.E., Stamm A.M., Graybill J.R., Craven P.C., Stevens D.A., Stiller R.L., Sarosi G.A., Medoff G., Gregg C.R., Gallis H.A., Fields B.T. Jr., Marier R.L., Kerkering T.A., Kaplowitz L.G., Cloud G., Bowles C. \& Shadomy S. 1983. Treatment of systemic mycoses with ketoconazole: emphasis on toxicity and clinical response in 52 patients. National Institute of Allergy and Infectious Diseases collaborative antifungal study. Annals of Internal Medicine. 98: 13-20.

19 Dunstan R.W., Langham R.F., Reimann K.A. \& Wakenell P.S. 1986. Feline sporotrichosis: a report of five cases with transmission to humans. Journal of the American Academy of Dermatology. 15: 37-45.

20 Dunstan R.W., Reimann K.A. \& Langham R.F. 1986. Feline sporotrichosis. Journal of the American Veterinary Medical Association. 189: 880-883.

21 Fernandes C.G.N., Moura S.T., Dantas A.F.M. \& Blatt M.C.S. 2004. Esporoticose felina - aspectos clínico-epidemiológicos: relato de casos (Cuiabá, Mato Grosso, Brasil). Revista Científica de Medicina Veterinária - Pequenos Animais e Animais de Estimação. 2: 39-43.

22 Ferrer L., Ramos J.A., Bonavia R., Cabañes J. \& Pumarola M. 1992. Cryptococcosis in two cats seropositive for feline immunodeficiency virus. Veterinary Record. 131: 393-394.

23 Fillipin F.B. \& Souza L.C. 2006. Eficiência terapêutica das formulações lipídicas de anfotericina B. Revista Brasileira de Ciências Farmacêuticas. 42: 167-194.

24 Findlay G.H., Vismer H.F. \& Dreyer L. 1984. Studies on sporotrichosis. Pathogenicity and morphogenesis in the Transvaal strains of Sporothrix schenckii. Mycopathologia. 87: 85-93.

25 Francesconi G., Valle A., Passos S., Reis R. \& Galhardo M. 2009. Terbinafine ( $250 \mathrm{mg} /$ day): an effective and safe treatment of cutaneous sporotrichosis. Journal of the European Academy of Dermatology and Venereology. [in press].

26 Freitas D., Migliano M. \& Zani Neto L. 1956. Esporotricose - Observação de caso espontâneo em gato doméstico ( $F$. catus). Revista da Faculdade de Medicina Veterinária de São Paulo. 5: 601-604.

27 Galhardo M.C., De Oliveira R.M., ValleA.C., Paes R. A., Silvatavares P.M., Monzon A., Mellado E., Rodriguez-Tudela J.L. \& Cuenca-Estrella M. 2008. Molecular epidemiology and antifungal susceptibility patterns of Sporothrix schenckii isolates from a cat-transmitted epidemic of sporotrichosis in Rio de Janeiro, Brazil. Medical Mycology. 46: 141-51.

28 Gougerot H. 1950. New insight gained in general pathology and practical medicine by the study of sporotrichoses. Annals of the New York Academy of Sciences. 50: 1348-1356.

29 Greene C.E. 2006. Antifungal chemotherapy. In: C.E. Greene (Eds). Infectious Diseases of the Dog and Cat. Philadelphia: Saunders Elsevier, pp.542-50. 
30 Gremião I.D.F., Pereira S.A., Rodrigues A.M., Figueiredo F.B., Nascimento Jr. A., Santos I.B. \& Schubach T.M.P. 2006. Tratamento cirúrgico associado à terapia antifúngica convencional na esporotricose felina. Acta Scientiae Veterinariae. 34: 221-223.

31 Gremião I.D.F., Schubach T.M.P., Pereira S.A., Rodrigues A.M., Chaves A.R. \& Barros M.B.L. 2009. Case report: intralesional amphotericin b in a cat with refractory localised sporotrichosis. Journal of Feline Medicine and Surgery. [in press].

32 Haruna K., Shiraki Y., Hiruma M., Ikeda S. \& Kawasaki M. 2006. A case of lymphangitic sporotrichosis occurring on both forearms with a published work review of cases of bilateral sporotrichosis in Japan. The Journal of Dermatology. 33: 364-367.

33 Hay R.J. 1999. Therapeutic potential of terbinafine in subcutaneous and systemic mycoses. British Journal of Dermatology. 141 (Suppl 56): 36-40.

34 Heit M.C. \& Riviere J. 1995. Antifungal and Antiviral Drugs. In: R. Adams (Eds). Veterinary Pharmacology and Therapeutics. Ames: Iowa State University Press, pp.855-885.

35 Hirano M., Watanabe K., Murakami M., Kano R., Yanai T., Yamazoe K., Fukata T. \& Kudo T. 2006. A case of feline sporotrichosis. The Journal of Veterinary Medical Science. 68: 283-284.

36 Hiruma M. \& Kagawa S. 1983. The effects of heat on Sporothrix schenckii in vitro and in vivo. Mycopathologia. 84: 21-30.

37 Hiruma M. \& Kagawa S. 1986. Effects of hyperthermia on phagocytosis and intracellular killing of Sporothrix schenckii by polymorphonuclear leukocytes. Mycopathologia. 95: 93-100.

38 Hiruma M., Katoh T., Yamamoto I. \& Kagawa S. 1987. Local hyperthermia in the treatment of sporotrichosis. Mykosen. 30: $315-321$.

39 Honse C.O., Rodrigues A.M., Gremião I.D.F., Pereira S.A. \& Schubach T.M. 2009. Use of local hypertermia to treat sporotrichosis in a cat. Veterinary Record. [in press].

40 Hull P.R. \& Vismer H.F. 1992. Treatment of cutaneous sporotrichosis with terbinafine. British Journal of Dermatology. 126 (Suppl 39): 51-55.

41 Jesus J.R. \& Marques S.M.T. 2006. Esporotricose cutânea em um gato - relato de caso. Clínica Veterinária. 65: $72-74$.

42 Jung J.Y., Almond C.H., Campbell D.C., Elkadi A. \& Tenorio A. 1979. Role of surgery in the management of pulmonary sporotrichosis. The Journal of Thoracic and Cardiovascular Surgery. 77: 234-239.

43 Kauffman C.A. 1995. Old and new therapies for sporotrichosis. Clinical Infectious Diseases. 21: 981-985.

44 Kauffman C.A., Bustamante B., Chapman S.W. \& Pappas P.G. 2007. Clinical practice guidelines for the management of sporotrichosis: 2007 update by the Infectious Diseases Society of America. Clinical Infectious Diseases. 45: 1255-1265.

45 Kauffman C.A., Hajjeh R. \& Chapman S.W. 2000. Practice guidelines for the management of patients with sporotrichosis. For the Mycoses Study Group. Infectious Diseases Society of America. Clinical Infectious Diseases. 30: 684-687.

46 Kohler L.M., Monteiro P.C., Hahn R.C. \& Hamdan J.S. 2004. In vitro susceptibilities of isolates of Sporothrix schenckii to itraconazole and terbinafine. Journal of Clinical Microbiology. 42: 4319-4320.

47 Lyman C.A. \& Walsh T.J. 1992. Systemically administered antifungal agents. A review of their clinical pharmacology and therapeutic applications. Drugs. 44: 9-35.

48 MacDonald E., Ewert A. \& Reitmeyer J.C. 1980. Reappearance of Sporothrix schenckii lesions after administration of Solu-Medrol to infected cats. Sabouraudia. 18: 295-300.

49 Mackay B.M., Menrath V.H., Ridley M.F. \& Kelly W.R. 1986. Sporotrichosis in a cat. Australian Veterinary Practitioner. 16: 3-5.

50 Madrid I.M., Mattei A., Martins A., Nobre M. \& Meireles M. 2009. Feline Sporotrichosis in the Southern Region of Rio Grande do Sul, Brazil: Clinical, Zoonotic and Therapeutic Aspects. Zoonoses and Public Health. [in press].

51 Malik R., Craig A.J., Wigney D.I., Martin P. \& Love D.N. 1996. Combination chemotherapy of canine and feline cryptococcosis using subcutaneously administered amphotericin B. Australian Veterinary Journal. 73: 124-128.

52 Malik R., Wigney D.I., Muir D.B., Gregory D.J. \& Love D.N. 1992. Cryptococcosis in cats: clinical and mycological assessment of 29 cases and evaluation of treatment using orally administered fluconazole. Journal of Medical and Veterinary Mycology. 30: 133-144.

53 Marani L. \& Venturi S. 1986. Iodio e immunita ritardata. Minerva Medica. 77: 805-809.

54 Marimon R., Serena C., Gene J., Cano J. \& Guarro J. 2008. In vitro antifungal susceptibilities of five species of Sporothrix. Antimicrobial Agents and Chemotheraphy. 52: 732-734. 
55 Marques S.A., Franco S.R., Camargo R.M., Dias L.D., Haddad Junior V. \& Fabris V.E. 1993. Sporotrichosis of the domestic cat (Felis catus): human transmission. Revista do Instituto de Medicina Tropical de São Paulo. 35: $327-330$.

56 Martinez R. 2006. Atualização no uso de agentes antifúngicos. Jornal Brasileiro de Pneumologia. 32: 449-460.

57 Medleau L., Marks M.A., Brown J. \& Borges W.L. 1990. Clinical evaluation of a cryptococcal antigen latex agglutination test for diagnosis of cryptococcosis in cats. Journal of the American Veterinary Medical Association. 196: $1470-1473$.

58 Meinerz A.R., Nascente P.S., Schuch L.F., Cleff M.B., Santin R., Brum C.S., Nobre M. O., Meireles M.C. \& Mello J.R. 2007. Susceptibilidade in vitro de isolados de Sporothrix schenckii frente a terbinafina e itraconazol. Revista da Sociedade Brasileira de Medicina Tropical. 40: 60-62.

59 Naqvi S.H., Becherer P. \& Gudipati S. 1993. Ketoconazole treatment of a family with zoonotic sporotrichosis. Scandinavian Journal of Infectious Diseases. 25: 543-545.

60 Nobre M.O., Castro A.P., Caetano D., Souza L.L., Meireles M.C.A. \& Ferreiro L. 2001. Recurrence of sporotrichosis in cats with zoonotic involvement. Revista Iberoamericana de Micologia. 18: 137-140.

61 Nobre M.O., Nascente P.S., Meireles M.C. \& Ferreiro L. 2002. Drogas antifúngicas para pequenos e grandes animais. Ciência Rural. 32: 175-184.

62 Nusbaum B.P., Gulbas N. \& Horwitz S.N. 1983. Sporotrichosis acquired from a cat. Journal of American Academy of Dermatology. 8: 386-391.

63 Papich M. 1989. Effect of drugs on pregnancy. In: Bonagura J. \& Kirk R. (Eds). Current Veterinary Therapy X. Philadelphia: Saunders, pp.1291-1299.

64 Pereira S.A., Passos S.R.L., Silva J.N., Gremião I.D.F., Figueiredo F.B., Teixeira J.L., Monteiro P.C.F. \& Schubach T.M. 2009. Therapeutic response to azolic anti fungal agents for treating feline sporotrichosis. Veterinary Record. [in press].

65 Pereira S.A., Schubach T.M.P., Figueiredo F.B., Leme L.R.P., Santos I.B., Okamoto T., Cuzzi T., Reis R.S. \& Schubach A. 2005. Demodicose associada à esporotricose e pediculose em um gato co-infectado por FIV/FeLV. Acta Scientiae Veterinariae. 33: 75-78.

66 Perez A. 1999. Terbinafine: broad new spectrum of indications in several subcutaneous and systemic and parasitic diseases. Mycoses. 42 (Suppl 2): 111-4.

67 Pinto O.M.F.C. 2006. Influência, in vitro, da vancomicina, da anfotericina B desoxicolato e de ambas sobre a unção fagocitária, produção de radicais de oxigênio e fator de necrose tumoral por fagócitos de sangue periférico de indivíduos normais. 135f. Brasília - DF. Dissertação (Mestrado em Medicina Tropical) Programa de Pós-graduação em Medicina Tropical, Universidade de Brasília.

68 Plumb D.C. 2006. Manual de Farmacología Veterinaria. 5.ed. Buenos Aires: Inter-Médica, 870p.

69 Restrepo A., Robledo J., Gomez I., Tabares A.M. \& Gutierrez R. 1986. Itraconazole therapy in lymphangitic and cutaneous sporotrichosis. Archives of Dermatology. 122: 413-417.

70 Rippon J. 1988. Sporotrichosis. In: Rippon J. (Ed). Medical Mycology - The Pathogenic Fungi and the Pathogenic Actinomycetes. Philadelphia: W.B. Saunders Company, pp.325-352.

71 Rocha M.F.G. \& Sidrim J.J.C. 1999. Drogas antifúngicas. In: Sidrim, J. J. C \& Moreira, J. L. B. (Eds). Fundamentos clínicos laboratoriais da micologia médica. 1.ed. Rio de Janeiro: Guanabara Koogan, pp.36-44.

72 Rodrigues A.M. 2009. Anfotericina B subcutânea associada ao itraconazol no tratamento da esporotricose em gatos domésticos. 70f. Rio de Janeiro - RJ. Dissertação (Mestrado em Pesquisa Clínica em Doenças Infecciosas) Programa de Pós-graduação em Pesquisa Clínica em Doenças Infecciosas, Fundação Oswaldo Cruz.

73 Rosser E. \& Dunstan R. 2006. Sporotrichosis. In: Greene C.E. (Ed). Infectious diseases of the dog and cat. Philadelphia: Saunders Elsevier, 1424pp.

74 Samorodin C.S. \& Sina B. 1984. Ketoconazole-treated sporotrichosis in a veterinarian. Cutis. 33: 487-488.

75 Schell W. 1998. Agents of chromoblastomycosis and sporotrichosis. In: L. Ajello L. \& Hay R.J. (Eds). Topley \& Wilson'sMicrobiology and Microbial Infections. London: Arnold, pp.315-336.

76 Schubach A., Barros M.B. \& Wanke B. 2008. Epidemic sporotrichosis. Current Opinion in Infectious Diseases. 21: $129-133$.

77 Schubach T.M., Schubach A., Okamoto T., Barros M.B., Figueiredo F.B., Cuzzi T., Fialho-Monteiro P.C., Reis R.S., Perez M.A. \& Wanke B. 2004. Evaluation of an epidemic of sporotrichosis in cats: 347 cases (1998-2001). Journal of the American Veterinary Medical Association. 224: 1623-1629. 
78 Schubach T.M., Schubach A., Okamoto T., Barros M.B., Figueiredo F.B., Cuzzi T., Pereira S.A., Santos I.B., Almeida Paes R., Paes Leme L.R. \& Wanke B. 2006. Canine sporotrichosis in Rio de Janeiro, Brazil: clinical presentation, laboratory diagnosis and therapeutic response in 44 cases (1998-2003). Medical Mycology. 44: 87-92.

79 Schubach T.M., Valle A.C., Gutierrez-Galhardo M.C., Monteiro P.C., Reis R.S., Zancope-Oliveira R.M., Marzochi K.B. \& Schubach A. 2001. Isolation of Sporothrix schenckii from the nails of domestic cats (Felis catus). Medical Mycology. 39: 147-149.

80 Scott D., Miller W. \& Griffin C. 1996. Doenças fúngicas da pele. In: Scott D., Muller G. \& Griffin C. (Eds). Muller \& Kirk - Dermatologia de pequenos animais. Rio de Janeiro: Interlivros Edições Ltda, pp.301-369.

81 Shinogi T., Misago N. \& Narisawa Y. 2004. Cutaneous sporotrichosis with refractory and reinfectious lesions in a healthy female. Journal of Dermatology. 31: 492-496.

82 Silva D.T., Pereira S.A., Gremião I.D.F., Chaves A.R., Cavalcanti M.C.H., Silva J.N. \& Schubach T.M. 2008. Esporotricose conjuntival felina. Acta Scientiae Veterinariae. 36: 181-184.

83 Singer J.I. \& Muncie J.E. 1952. Sporotrichosis: etiologic considerations and report of additional cases from New York. New York State Journal of Medicine. 52: 2147-2153.

84 Sykes J.E., Torres S.M., Armstrong P.J. \& Lindeman C.J. 2001. Itraconazole for treatment of sporotrichosis in a dog residing on a Christmas tree farm. Journal of the American Veterinary Medical Association. 218: 1440-1443.

85 Taboada J. 2000. Systemic mycoses. In: Ettinger S. \& Feldman E. (Eds). Textbook of veterinary internal medicine Diseases of the dog and cat. Philadelphia: W. B. Saunders Company, pp.453-476.

86 Wada R. 1968. Studies on mode of action of potassium iodide upon sporotrichosis. Mycopathologia et Mycologia Applicata. 34: 97-107.

87 Welsh R.D. 2003. Sporotrichosis. Journal of the American Veterinary Medical Association. 223: 1123-1126.

88 Willard M.D., Nachreiner R.F., Howard V.C. \& Fooshee S.K. 1986. Effect of long-term administration of ketoconazole in cats. American Journal of American Veterinary Research. 47: 2510-2513. 